\title{
Variabilité des phénomènes d'interférence entre Vicia sativa L. et Avena sativa L.. I. Dynamique de croissance de la vesce dans un peuplement associé de vesce-avoine
}

\author{
M. Ouknider * et P. Jacquard \\ Unité de biologie des populations et des peuplements, CNRS, BP 5051, 34033 Montpellier, France
}

(reçu le 17-8-1988, accepté le 1-2-1989)

Résumé - Ce travail analyse la variation de la surface foliaire et de l'indice foliaire sous l'influence de la densité de peuplement et de la fertilisation azotée. Le paramètre surface foliaire a réagi différemment chez les deux espèces aux variables étudiées. La fertilisation azotée, ayant augmenté la croissance de l'avoine, a exercé un effet dépressif sur la croissance de la vesce.

Une relation entre l'indice foliaire global et la matière sèche produite par les parties aériennes, en association, a été mise en évidence. Toujours en association, la compétition pour la lumière a été étudiée à partir de l'analyse de la structure des profils de surface foliaire, d'une part, et par la contribution de la vesce à l'indice foliaire total, d'autre part. II se dégage de cela que la compétition pour la lumière est non seulement déterminée par la position des feuilles dans les différentes strates du couvert, mais aussi par la contribution de chaque espèce à l'indice foliaire global.

association vesce-avoine - compétition pour la lumière - croissance - profil de surface foliaire - indice foliaire

Summary - Variability of the phenomena of interferences between Vicia sativa L. and Avena sativa L. I. Growth dynamics of the vetch in a mixed stand of vetch and oat. The work analyses the variation of the leaf area and of the leaf area index (LAI) under the effects of stand density and nitrogen supply. The leaf area parameter has differentially responded for the 2 species to the studied variables. The nitrogen fertilization, increasing the growth of oat, has determined a depressive effect on the growth of vetch.

A relationship between the global LAl and the biomass (DM) of the shoots, in mixture, has been evidenced. In mixture, the competition for light has been studied with an analysis of the structure of the leaf area profiles, on one hand, and, on the other hand, through the contribution of the vetch to the global LAI. It results that the competition for light is not only induced by the position of the leaves in the several strata of the cover, but also by the contribution of each species to the global LAI.

vetch-oat mixtures - competition for light - growth - leaf area profile - leaf area index

\section{Introduction}

Les associations graminées-légumineuses, en dehors d'une meilleure répartition de leur production par rapport aux cultures pures, se justifient surtout par des avantages nutritionnels, dont les économies d'engrais azoté nécessaire à une graminée (Laissus, 1979; Haynes, 1980).

Des productions équivalentes à celle de la graminée en culture pure ont été souvent rappor- tées (Plancquaert, 1976; Arnaud et Niqueux, 1982; Martin et Snaydon, 1982). Haynes (1980) a signalé que l'association est toujours plus productive que la culture pure de la graminée sans apport d'azote. Toutefois, la conduite d'une association est toujours difficile, car il faut maintenir un équilibre entre deux constituants très différents. L'azote est un élément déterminant dans les relations entre les deux partenaires. Des apports d'azote sur l'association se traduisent par

*Adresse actuelle: Département d'agronomie et d'amélioration des plantes, ENA, BPS 40, Meknès, Maroc. 
une augmentation de la participation de la graminée à la biomasse totale et par une diminution de la contribution de la légumineuse. La fertilisation azotée accentue la compétition pour la lumière entre les 2 espèces, en augmentant la croissance de l'espèce dominante.

Lorsqu'une légumineuse est associée à une graminée, son assimilation d'azote est limitée et, si elle n'est pas dominée, elle fixera plus d'azote atmosphérique. Chalamet et al. (1983), sur trèfle violet associé au ray-grass d'Italie, ont montré que l'association est favorable à la fixation et défavorable à l'assimilation. Alors que dans le cas de la luzerne pure, le pic d'ARA (activité réductrice d'acétylène) spécifique ne change pas entre la première et la deuxième repousse, il augmente dans le cas de l'association avec le dactyle (Wery, 1983). Cet effet favorable peut résulter des prélèvements d'azote minéral par les graminées et ne se traduit pas toujours par une amélioration des quantités fixées, qui dépendent du pouvoir de compétition de la légumineuse. Dans le cas de partenaires pour lesquels la compétition est beaucoup plus précoce, l'association diminue même la fixation tout en réduisant l'énergie disponible pour les nodosités. Valis (1978) a signalé que dans certains cas les légumineuses peuvent devenir elles aussi très agressives dans la compétition. Sur l'association luzerne-dactyle, Cruz (1985) et Cruz et Lemaire (1986) ont montré qu'en présence d'apport d'azote, la luzerne diminue le niveau de nutrition azotée du dactyle. II semblerait, selon ces auteurs, que lorsque la disponibilité en azote minéral du sol augmente, la luzerne devienne compétitive vis-à-vis du dactyle pour son utilisation. En revanche, Faris et Ta (1985) ont indiqué que la luzerne a un effet bénéfique sur la productivité de la graminée associée (Phleum pratense). Jacquard (1977) concluait d'ailleurs que, dans les associations où la légumineuse est représentée par la luzerne, des résultats contradictoires ont été obtenus.

L'effet des graminées sur les légumineuses est variable selon les espèces associées (parfois très marqué). En revanche, l'effet des légumineuses sur les graminées est manifeste. Les apports d'azote par les nodosités des légumineuses ont pu être mis en évidence dans de nombreux essais, même si leur ampleur et les modes d'action sont encore mal connus et posent des problèmes d'ordre expérimental. Le potentiel de production des graminées en association est supérieur à celui des graminées en culture pure. II en est de même pour la teneur en azote total. Le transfert d'azote de la légumineuse vers la graminée, évoqué par plusieurs auteurs, s'appuie dans certains travaux sur les teneurs en azote total de la graminée associée
(Doberti, 1974; Charles, 1982; Broadbent et al., 1982; Arnaud et Niqueux, 1982). Cette interprétation basée uniquement sur les teneurs en azote total a été contestée par Cruz (1985) et Cruz et Lemaire (1986), qui ont développé une approche nouvelle fondée sur les courbes de dilution de l'azote dans la matière sèche au cours de la croissance, processus principalement décrit et discuté dans le cas de cultures de graminées (Salette et Lemaire, 1981; Lemaire et Salette, 1984). La méthode a été appliquée aux peuplements associés (luzerne-dactyle) par Wery (1983), Cruz (1985), Cruz et Lemaire (1986). Ces derniers auteurs ont conclu qu'en l'absence d'apport d'azote les augmentations de teneurs en azote constatées sont entièrement explicables par l'effet dépressif de la luzerne sur la croissance du dactyle, et qu'il ne s'agit pas d'une amélioration de sa nutrition azotée. Des travaux récents basés sur l'utilisation de l'azote marqué (N15) ont démontré l'existence d'un transfert d'azote de la légumineuse vers la graminée avec plusieurs espèces de légumineuses (Lescure et al., 1986; Ismaili et Weaver, 1986; Ta et Faris, 1987) et en solutions nutritives, en hydroponie, par Wacquant et al. (1981). L'existence de transferts inverses, des graminées vers les légumineuses, a été également avancée.

L'objectif de ce travail est de déterminer l'effet d'un apport d'azote sur les relations entre les deux composants de l'association en liaison avec la densité de peuplement. La croissance et la biomasse aérienne de l'association sont étudiées dans cet article; la nutrition azotée sera présentée dans un second article.

\section{Matériel et Méthodes}

\section{Facteurs étudiés}

Parmi les facteurs susceptibles de modifier la surface foliaire d'un couvert végétal, 2 ont été retenus: la densité de peuplement et la fertilisation azotée. Trois densités de peuplement ont été étudiées: 150,300 et 450 individus par $\mathrm{m}^{2}$, combinées à 3 doses d'azote: 0,60 et 120 unités par hectare. Ces variables ont été testées sur l'association et les cultures pures correspondantes de vesce et d'avoine. Le matériel végétal est constitué d'une vesce: variété 6242 et d'une avoine: variété 153 tutrice.

\section{Techniques culturales}

Après la récolte du précédent cultural (blé), la parcelle d'essais a été l'objet d'un labour profond (9-8-1986), suivi d'un passage croisé de cover-crop. La fertilisation phosphopotassique composée de 100 unités de $\mathrm{P}_{2} \mathrm{O}_{5}$ et 100 unités de $\mathrm{K}_{2} \mathrm{O}$ a été enfouie par un deuxième passage croisé (31-11-1986) qui sert en même temps à préparer le lit de semence. Le semis a eu lieu le 5 décembre 1986 et a été réalisé manuellement. Pour l'association, les graines de vesce et d'avoine sont 
localisées sur la même ligne, la proportion était de 50V-50A ( $V=$ vesce; $A=$ avoine). L'écartement entre les lignes est constant $(0,20 \mathrm{~m})$ et sur la ligne celui-ci est variable selon la densité testée.

La fertilisation azotée a été épandue le 19 janvier 1987. L'expérience a été irriguée 2 fois et maintenue propre par des désherbages manuels. La récolte a été effectuée le 30 avril, alors que l'avoine était au stade de pleine épiaison et la vesce au stade de formation des gousses. Le rendement a été estimé sur $2 \times 1 \mathrm{~m}^{2}$ pour chacun des traitements.

\section{Dispositif expérimental}

Le dispositif adopté comporte les combinaisons des différents niveaux de facteurs étudiés, chacune est attribuée à une parcelle de $4 \times 2 \mathrm{~m}^{2}$. II s'agit d'un dispositif en blocs randomisés. L'essai comporte 4 blocs de 27 parcelles élémentaires. Les blocs sont séparés par des allées de $2 \mathrm{~m}$ et les parcelles par des allées de $1 \mathrm{~m}$.

\section{Modalités et fréquences des prélèvements}

La croissance en matière sèche a été suivie au cours du cycle par des prélèvements de biomasse, sur des placettes de 0,25 mètre linéaire pour chaque traitement et sur 3 blocs.

Sur chaque prélèvement, on détermine le poids de la biomasse fraîche et sèche. Le broyat de chaque échantillon obtenu après séchage $\left(80^{\circ} \mathrm{C}\right.$ pendant $\left.48 \mathrm{~h}\right)$ a été utilisé pour le dosage d'azote total selon la méthode de Kjeldahl. Un autre prélèvement, de même taille, sur le 4 e bloc est fait simultanément sur chaque traitement et sert à déterminer d'une part la richesse en feuilles caractérisée par le rapport feuilles sur tiges $(F / T)$ et, d'autre part, les autres paramètres de croissance et de développement. Six plantes sont tirées au hasard (12 pour l'association, 6 par espèce) sur lesquelles le nombre de tiges par plante, le nombre de feuilles totales, la hauteur et la surface foliaire sont déterminés, ainsi que le poids des feuilles et des tiges (frais et sec). Les surfaces foliaires sont mesurées par un analyseur d'images automatique. La fréquence des prélèvements a varié entre une semaine et 10 jours.

\section{Exploitation des résultats}

On a appliqué aux résultats obtenus une analyse de variance avec interaction lorsque les paramètres observés sont expliqués par les facteurs étudiés. Les tests de signification ont été réalisés à l'aide du F de Snedecor et la comparaison des moyennes par la méthode de Newman \& Keuls.

\section{Résultats et discussion}

\section{Biomasse sèche aérienne par plante}

Les courbes de la Figure 1a permettent d'analyser la dynamique de croissance de l'avoine à 2 niveaux d'azote ( 0 et $60 \mathrm{u}$.) dans les peuplements purs $\left(d_{1}\right.$ et $\left.d_{2}\right)$ et dans le peuplement $d_{2}(300$ ind. $/ \mathrm{m}^{2}$ ) pour l'association. Cela permet de comparer la croissance de l'avoine associée en peuplement $d_{2}$, à même densité numérique (150 ind. $\left./ \mathrm{m}^{2}\right)$, à celle de l'avoine pure en $d_{1}$, à 2
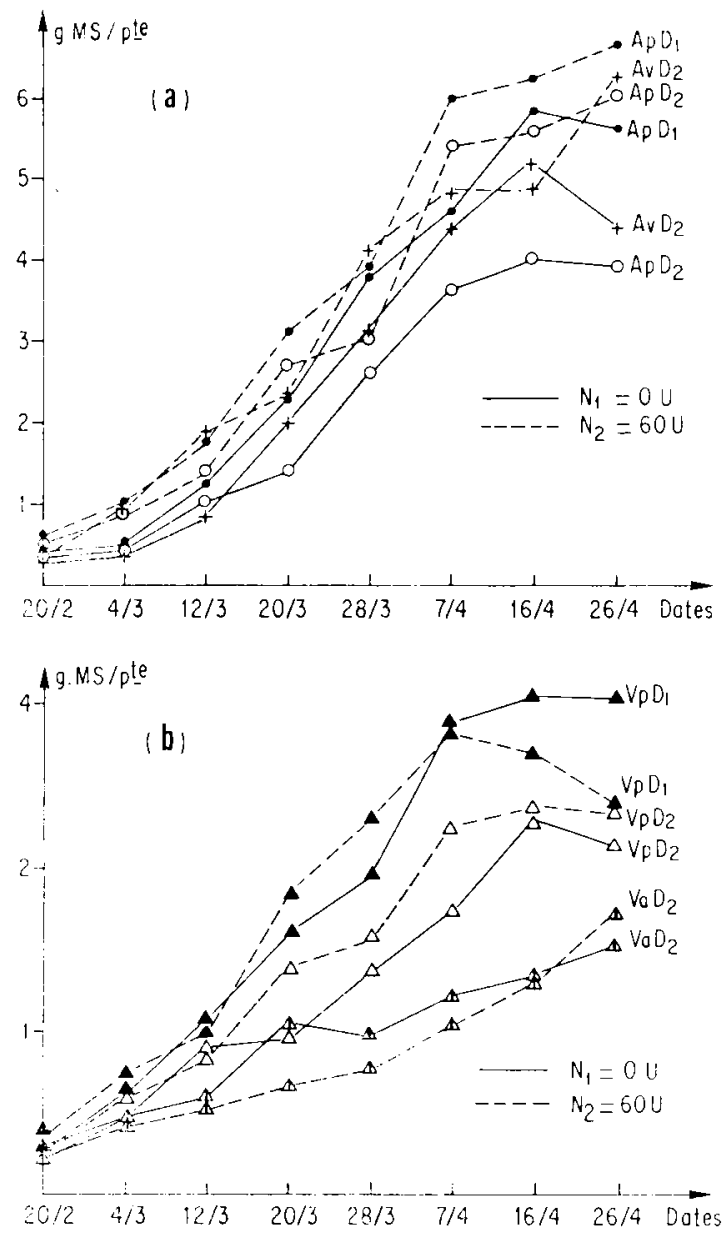

Fig. 1. Evolution de la biomasse sèche aérienne par plante d'avoine (a) et de vesce (b) en fonction du temps. Variation avec le niveau d'azote ( type de culture (culture pure et association) et la densité de peuplement.

ApD1: avoine pure à la densité $d_{1}: A_{2} D_{2}$ avoine pure à la densité $D_{2}: O ; A \vee D_{2}$ : avoine associée à la densité $D_{2}:+$; VpD1: vesce pure à la densité $D_{1}: \Delta ; V_{p} D_{2}$ : vesce pure à la densité $\mathrm{D}_{2}: \Delta ; V \mathrm{~V}_{2}$ : vesce associée à la densité $\mathrm{D}_{2}: \Delta ; D_{1}=$ 150 ind.$/ \mathrm{m}^{2} ; \mathrm{D}_{2}=300$ ind. $/ \mathrm{m}^{2}$.

niveaux d'azote, de manière à mettre en évidence l'effet de présence de la vesce sur son partenaire et par conséquent sur sa croissance. Ces courbes montrent que la vesce ne modifie pas la croissance de l'avoine; l'apport de 60 unités d'azote améliore la croissance de l'avoine pure par rapport à l'association non fertilisée.

Dans cette association, la croissance de l'avoine, à partir du stade montaison correspondant à la date du 12 mars est nettement améliorée par la présence de la vesce. Ceci confirme les résultats des essais antérieurs. (Ouknider et Jacquard, 1986; Ouknider et Jacquard, 1988).

Pour la vesce, la Figure $1 \mathrm{~b}$ montre qu'en culture pure la fertilisation améliore la croissance; toutefois, au faible peuplement $d_{1}$, la fertilisation azotée a eu un effet négatif sur la croissance de la vesce à partir du stade de début de floraison 
(7 avril). En association, la nutrition de la vesce est modifiée par la présence de l'avoine, l'effet dépressif est accentué par l'apport d'azote. L'azote ayant augmenté la croissance de l'avoine et par conséquent l'importance de l'ombrage des individus de vesce, il s'en suit que, dans ce cas, l'azote amplifie la compétition pour la lumière entre les 2 espèces. Cruz \& Lemaire (1986), sur l'association luzerne-dactyle, ont montré que la concurrence exercée par le dactyle sur la nutrition azotée de la luzerne reste toujours plus faible que l'effet dépressif de la luzerne vis-à-vis d'elle même. Selon ces auteurs, l'effet d'un apport d'azote sur le niveau de nutrition azotée des deux composants de l'association dépend en premier lieu de leur capacité respective de croissance. Ils ont mis en évidence que ce sont les différences relatives de croissance en début de repousse qui conditionnent la réponse de chacune des deux espèces associées à l'apport d'azote.

\section{Surface foliaire et indice foliaire}

Evolution de la surface foliaire de l'avoine et de la vesce

La surface foliaire par plante des 2 espèces (vesce et avoine) est fortement corrélée à la matière sèche produite par individu. II en est de même pour le poids spécifique des feuilles qui est corrélé à la matière sèche. Les résultats du
Tableau 1 regroupent les pentes des droites et les coefficients de corrélation obtenus pour la vesce et l'avoine en association à certains stades de développement (donnés à titre d'exemple).

Les courbes de la Figure 2 présentent l'évolution de la surface foliaire de l'avoine dans le cas de doses croissantes d'azote et pour chaque peuplement. Ces figures mettent en évidence le rôle déterminant de l'azote sur la surface foliaire qui est d'autant plus grande que la fertilisation azotée est augmentée. Une forte dose d'azote sur un peuplement faible $\left(d_{1}\right)$ conduit à la surface foliaire la plus élevée. Aux densités $d_{2}$ et $d_{3}$, aucun niveau d'azote ne permet de réaliser la surface foliaire permise par un niveau d'azote élevé sur un faible peuplement. La compétition pour la lumière a probablement limité l'expansion de la surface foliaire dans les peuplements $d_{2}$ et $d_{3}$. Pour les peuplements purs sans azote, la valeur plafond de la surface foliaire a été de 430 , 400 et $250 \mathrm{~cm}^{2} /$ plante pour $d_{1}, d_{2}$ et $d_{3}$. Pour les peuplements $d_{2}$ et $d_{3}$, en culture pure, le niveau d'azote (entre 0 et 120 unités) a moins d'influence $\left(+125 \mathrm{~cm}^{2}\right.$ et $+138 \mathrm{~cm}^{2}$ respectivement) sur la valeur maximale de la surface foliaire qu'au peuplement $d_{1}\left(+375 \mathrm{~cm}^{2}\right)$. Pour l'avoine associée, la valeur maximale de la surface foliaire augmente avec la fertilisation azotée, ce qui suggère que l'avoine dans l'association est à une densité biologique inférieure par rapport à sa culture pure. La densité numérique a affecté de

Tableau I. Caractéristiques des droites de régression de la biomasse sèche aérienne produite par plante de vesce et d'avoine, en association, en fonction du poids spécifique des feuilles (PS) d'une part et de la surface foliaire par plante d'autre part (SF). ( $n=27$ couples).

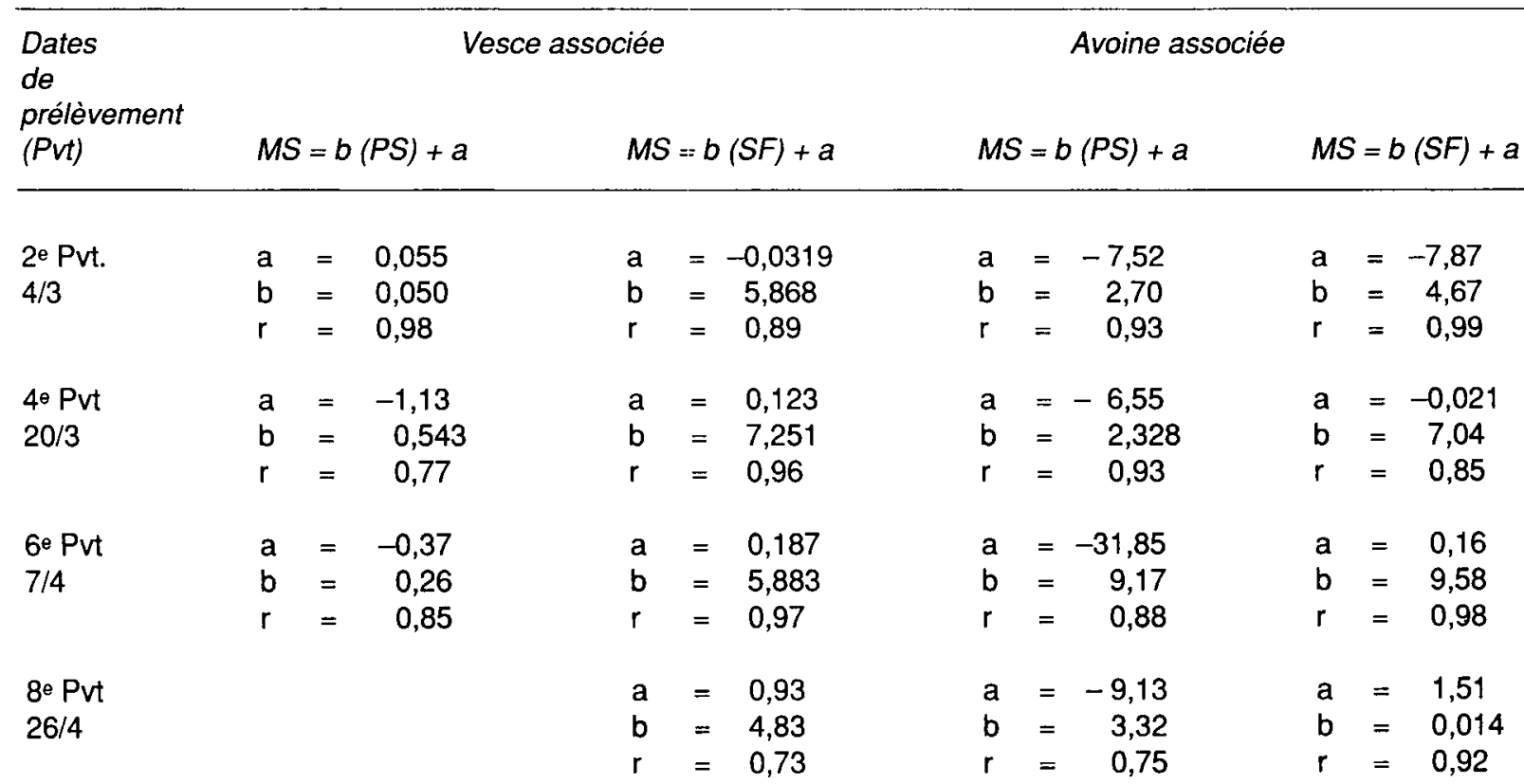

MS: matière sèche par plante (g); SF = surface foliaire ( $\mathrm{cm}^{2} /$ plante); PS: poids spécifique des feuilles $\left(\mathrm{mg} / \mathrm{cm}^{2}\right) ; \mathrm{r}$ : coefficient de corrélation; a et b: coefficients de l'équation: $M S=b x+a$. 

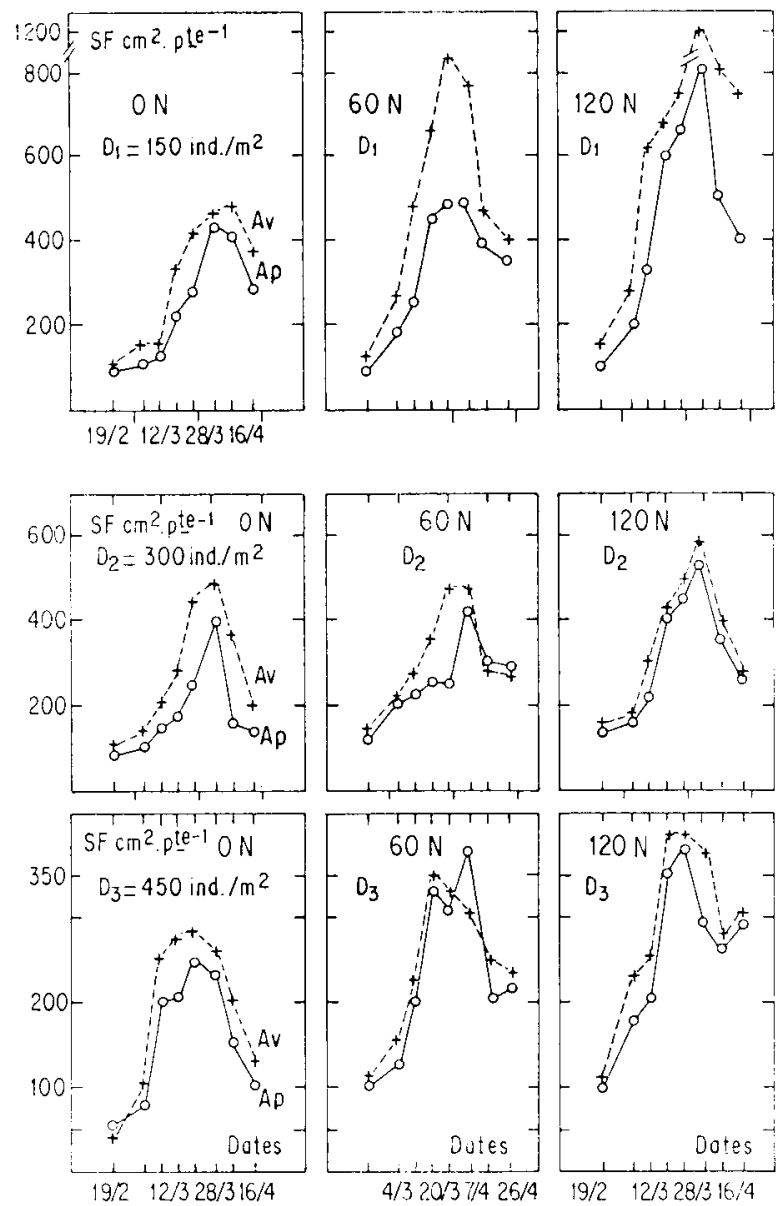

Fig. 2. Evolution de la surface foliaire par plant d'avoine en culture pure $(O)$ et en association $(x)$ en fonction du temps. Variation avec la densité de peuplement et le niveau d'azote. Ap: avoine pure; Av: avoine associée; D: densité; $N$ : niveau d'azote.

façon plus significative la valeur plafond de surface foliaire de l'avoine en peuplement pur et associé que la dose d'azote.

La Figure 3 permet d'analyser l'effet du partenaire avoine (compétition interspécifique), d'une part, et de la fertilisation azotée, d'autre part, sur la surface foliaire de la vesce et pour chacun des peuplements étudiés. Ces courbes montrent que, sur les peuplements purs, la fertilisation azotée a un effet positif sur la surface foliaire de la vesce. Toutefois, à densité moyenne $d_{2}$ et à forte densité $d_{3}$, le niveau d'azote 120 unités a un effet négatif; le passage de 60 à 120 unités d'azote n'a pas modifié la valeur plafond de surface foliaire. En association, quelle que soit la densité, la fertilisation azotée a un effet dépressif sur la surface foliaire de la vesce au stade épiaison de la graminée, correspondant à la date du 16/4; pour le peuplement $d_{2}$ la surface foliaire de la vesce a varié de 175,150 et $100 \mathrm{~cm}^{2} /$ plante respectivement pour 0,60 et 120 unités d'azote. L'apport d'azote a engendré une diminution de la surface foliaire de $25 \%$ et $43 \%$ respectivement
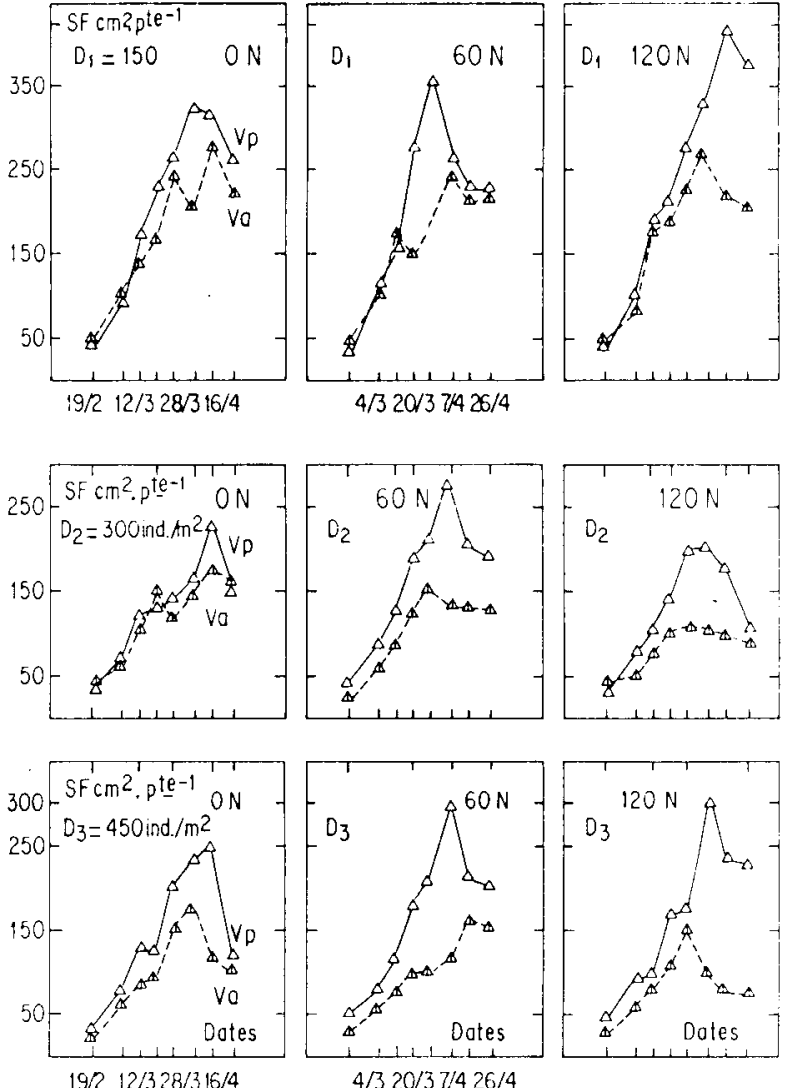

Fig. 3. Evolution de la surface foliaire par plant de vesce en culture pure $(\Delta)$ et en association $(\Delta)$ en fonction du temps. Variation avec la densité de peuplement et le niveau d'azote. $\mathrm{Vp}$ : vesce pure; Va: vesce associée; D: densité; $N$ : niveau d'azote.

pour 60 et 120 unités d'azote. En peuplement dense $\left(d_{3}\right)$ l'apport d'azote, 60 ou 120 unités, a engendré la même perte de $25 \%$.

Les profils de surface foliaire de la vesce et de l'avoine en culture associée

Pour analyser les phénomènes d'ombrage exercés par l'avoine, nous avons déterminé, à partir du stade fin montaison, la surface foliaire par tranche de $20 \mathrm{~cm}$ de hauteur pour les deux espèces en association, ce qui nous a permis de tracer les profils de surface foliaire correspondants et de voir si réellement il y a une compétition pour la lumière. Les Figures 4 et 5 représentent les profils de surface foliaire aux stades de fin de montaison (28 mars) et au stade d'épiaison de la graminée (16 avril) pour les 3 peuplements étudiés et à 2 niveaux d'azote à chaque stade; 0 et 60 unités au stade de fin de montaison et 0 et 120 unités pour le stade d'épiaison.

Les hauteurs réelles développées par chacune des espèces dans le couvert mixte ont été prises au champ pour un stade donné, et la détermination de la surface foliaire par tranche a été faite au laboratoire en tenant compte de ces hauteurs. 

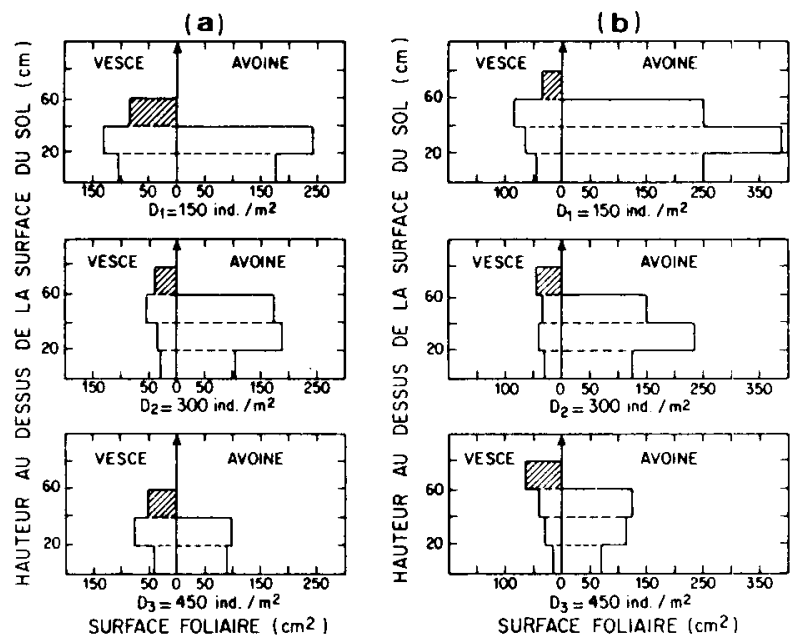

Fig. 4. Structure des profils de surface foliaire de la vesce et de l'avoine en association au stade fin montaison de la graminée. Variation avec la densité de peuplement (D) et le niveau d'azote (a: $N_{1}=0$ u.; b: $N_{2}=60$ u.).

La partie hachurée correspond à la strate supérieure de la vesce quand elle est dominante.
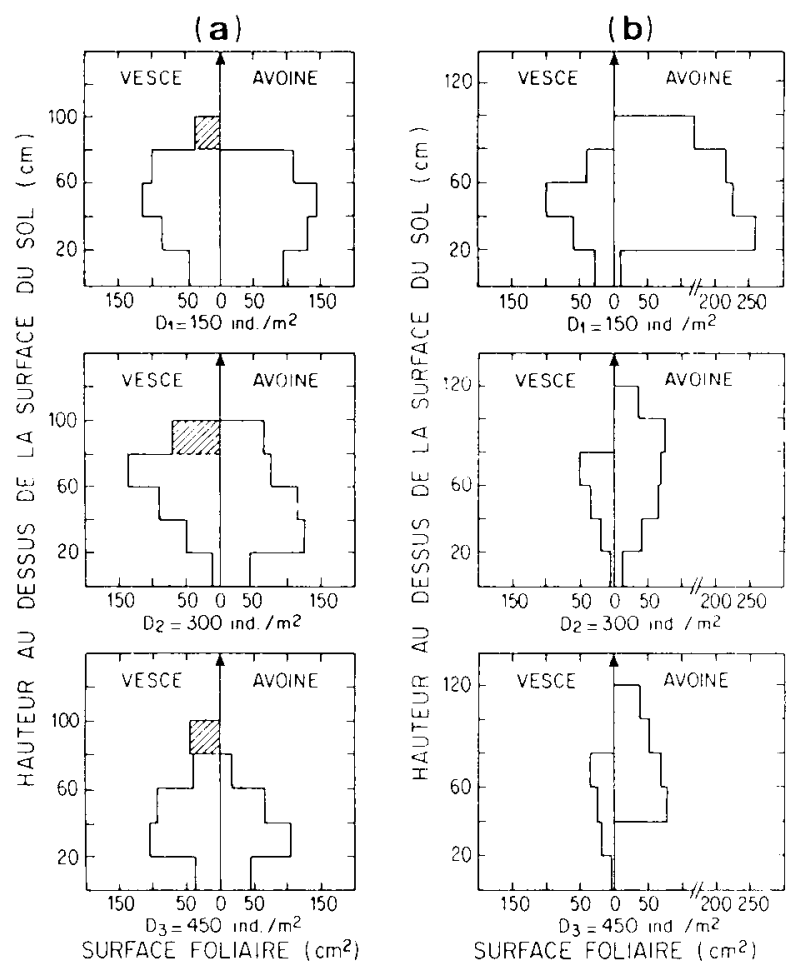

Fig. 5. Structure des profils de surface foliaire de la vesce et de l'avoine en association au stade épiaison de la graminée. Variation avec la densité de peuplement (D) et le niveau d'azote (a: $N_{1}=0$ u.; b: $N_{2}=120 u$.).

Sur la Figure 4, les profils de surface foliaire mettent en évidence un effet très net de la contrainte ombrage exercée par l'avoine. Au stade de fin de montaison de la graminée, la vesce en association, avec ou sans apport d'azote, se heurte à un manque de disponibilité en lumière. Dans toutes les densités testées, la vesce, pour échapper à la contrainte lumière, tend à occuper les strates supérieures où l'avoine est absente ou peu présente. La densité de la strate supérieure augmente, en valeur relative, avec la densité de peuplement en présence de fertilisation azotée. Pour l'association non fertilisée, le pourcentage de surface foliaire par rapport à la surface totale par plante se trouvant au dessus de l'avoine (strate $40-60 \mathrm{~cm}$ ) est de $27 \%$, $32 \%$ et $30 \%$ respectivement pour $d_{1}, d_{2}$ et $d_{3}$. Avec apport d'azote (60 unités), les individus de vesce s'étiolent davantage en occupant la couche supérieure $(60-80 \mathrm{~cm})$ dans laquelle le pourcentage de surface foliaire par rapport à la surface totale développée est de $19,5 \%, 37 \%$ et $66,4 \%$ respectivement pour $d_{1}, d_{2}$ et $d_{3}$; cela montre que la contrainte augmente en présence d'azote avec la densité de peuplement.

Au stade épiaison de la graminée (floraison vesce), correspondant à la date du 16 avril, soit 20 jours après, la structure des profils de surface foliaire est modifiée (Fig. 5). En absence d'azote, la vesce dans les peuplements $d_{1}$ et $d_{3}$ arrive à occuper la strate $80-100 \mathrm{~cm}$, où l'avoine est absente, soit un pourcentage de $11 \%$ et $15 \%$ respectivement pour $d_{1}$ et $d_{3}$, alors qu'en $d_{2}$ la state $80-100 \mathrm{~cm}$ est occupée par les deux espèces mais avec des proportions différentes: $36,6 \%$ pour la vesce et $18 \%$ seulement pour l'avoine. Avec apport d'azote (120 unités), la croissance de l'avoine est telle que la vesce n'arrive pas à atteindre la hauteur de son compétiteur.

La densité foliaire dans les couches inférieures $(20-40 \mathrm{~cm})$ et moyennes $(40-60 \mathrm{~cm})$ est très élevée au point que l'on assiste à une situation de dépérissement total des feuilles de la couche $0-20 \mathrm{~cm}$ pour les peuplements $d_{2}$ et $d_{3}$ chez les 2 espèces, la surface foliaire dans cette couche étant nulle. La modification de la structure des profils foliaires montre que l'intensité lumineuse diminue progressivement au fur et à mesure que la lumière pénètre dans le végétal, et cela est d'autant plus accentué que la densité de peuplement est augmentée et la fertilisation azotée élevée.

\section{Indice foliaire de l'association et contribution de la vesce à l'indice foliaire global}

Dans l'association, le phénomène de compétition pour la lumière a été approché par l'analyse de l'indice foliaire global en comparaison avec celui des peuplements purs, ainsi que par la contribution de la vesce dans chaque strate du couvert à l'indice foliaire global.

La surface foliaire permet une analyse de l'indice foliaire $\left(\mathrm{m}^{2}\right.$ de feuilles $/ \mathrm{m}^{2}$ de sol), lequel peut être un moyen pour déterminer l'intensité de 
la compétition pour la lumière dans la mesure où la vitesse d'occupation de l'espace aérien par une espèce est déterminante pour sa capacité à concurrencer l'autre espèce pour la lumière. En effet, la productivité d'un couvert végétal est en relation avec le rayonnement intercepté par ses organes photosynthétiques, lequel dépend essentiellement de l'indice foliaire (Gosse et al., 1984) et du rayonnement global.

L'évolution de l'indice foliaire, dans l'association et dans les peuplements purs correspondants pour deux niveaux de densités $d_{1}$ et $d_{2}$ et à 3 niveaux d'azote (Fig. 6), montre que l'indice foliaire de l'association est supérieur à celui des cultures pures. Pour une densité donnée, l'indice foliaire maximum (ou plafond) augmente avec la fertilisation azotée; au faible peuplement $\left(d_{1}\right)$ il est de 7,2, 11,2 et 12 respectivement pour 0,60 et $120 \mathrm{~N}$, alors qu'en $d_{2}$, dans le même ordre, l'indice foliaire maximum de l'association est de $8,2,10$ et 12 . En culture pure de vesce au peuplement $d_{1}$, l'indice foliaire maximum plafond est
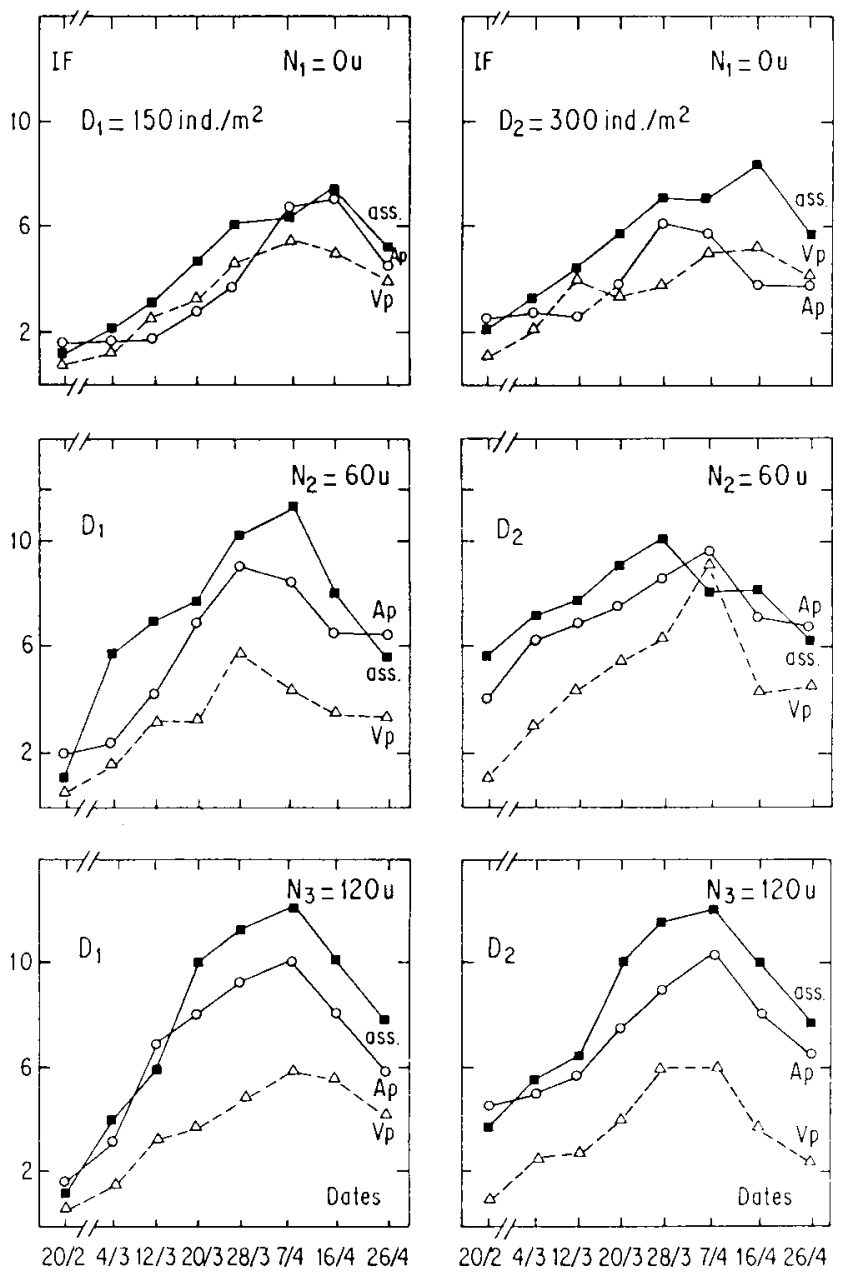

$20 / 24 / 312 / 320 / 328 / 37 / 416 / 426 / 4$

Fig. 6. Evolution de l'indice foliaire (IF) de l'avoine pure (Ap), de la vesce pure $(V p)$ et de leur association (ass.) en fonction du temps. Variation avec la densité de peuplement et le niveau d'azote.

$\mathrm{N}$ : niveau d'azote; D: densité; Avoine pure: (O); vesce pure $(\Delta)$; association (G). atteint pour des valeurs inférieures: $5,4,5,8$ et 5,8 respectivement pour 0,60 et $120 \mathrm{~N}$. II paraît être stable, alors que la surface foliaire et la matière sèche produite continuent à augmenter. Une telle situation a été décrite par Donald (1963) et rapportée par Brown et Blaser (1968).

A 120 unités d'azote, l'indice foliaire plafond n'est pas affecté par la densité de peuplement; il est de 12, 10 et 6 respectivement pour l'association, l'avoine pure et la vesce pure.

Des indices de surface foliaire supérieurs à ceux des cultures pures suggèrent une compétition pour la lumière comme cela été mis en évidence par les profils de surface foliaires. Ces résultats confirment ceux des essais antérieurs (Ouknider et Jacquard, 1988), dans lesquels l'effet proportion a été testé.

De même, les résultats du Tableau II vont dans le même sens. En absence d'azote, la contribution de la vesce à l'indice foliaire global est relativement importante 20 à $50 \%$. Selon les densités, elle diminue avec l'âge des plantes. Avec des niveaux d'azote élevés, la graminée devient dominante. Au stade d'épiaison de la graminée ( 7 avril), la contribution de la vesce à l'indice global est de $26,1 \%, 17,2 \%$ et $18,9 \%$ pour 60 unités d'azote et seulement de 15,3, 7,8 et $10,1 \%$ pour 120 unités respectivement pour $d_{1}, d_{2}$ et $d_{3}$. Cette contribution se situe essentiellement dans les strates inférieures et moyennes. Dans les strates les plus éclairées, ce pourcentage de contribution à l'indice foliaire global est faible et reste inférieur à $12 \%$. En l'absence d'azote la contribution de la strate supérieure $(60-80 \mathrm{~cm})$, à la densité $d_{2}$ n'est que de 5,1 et $6,5 \%$ respectivement aux stades montaison et épiaison. A faible densité $\left(d_{1}\right)$ la contribution de la strate supérieure passe de $11,6 \%$ pour la strate $40-60 \mathrm{~cm}$ au stade montaison à $3,1 \%$ au stade d'épiaison pour la strate $80-100 \mathrm{~cm}$. Avec apport de 120 u. d'azote, le pourcentage de contribution, dans la strate $60-80 \mathrm{~cm}$ où seule la vesce est présente n'est, au stade d'épiaison, que de 3,1 et $5,7 \%$ respectivement pour $d_{1}$ et $d_{2}$.

Dans le Tableau III, on a pondéré la contribution de la vesce (IFV) à l'indice foliaire global en fonction des tranches de profil occupées et de l'énergie interceptée $\left(R_{\mathrm{iv}}\right)$. Pour ce faire, on a tenu compte du fait que le rayonnement intercepté $\left(R_{i}\right)$ au niveau d'une strate était réduit par rapport au rayonnement reçu selon la loi de BeerLambert:

$\operatorname{Ln} \frac{(\mathrm{Ri})}{(\mathrm{Ro})}=-\mathrm{K} . \mathrm{IF}_{\mathrm{s}}$

avec:

$-R_{0}=$ rayonnement incident pris égal à l'unité, 
Tableau II. Contribution de la vesce à l'indice foliaire global de l'association (en \% du total).

\begin{tabular}{|c|c|c|c|c|c|c|c|c|c|}
\hline \multirow{2}{*}{$\begin{array}{l}\text { Azote } \\
\text { Dates de } \\
\text { prélèvement }\end{array}$} & \multicolumn{3}{|c|}{$N_{1}=O u$} & \multicolumn{3}{|c|}{$N 2=60 u$} & \multicolumn{2}{|c|}{$N_{3}=120 u$} & \multirow[b]{2}{*}{$d_{3}$} \\
\hline & $d_{1}$ & $d_{2}$ & $a_{3}^{\prime}$ & $d_{1}$ & $d_{2}$ & $d_{3}$ & $d_{1}$ & $d_{2}$ & \\
\hline $20-2$ & 45 & 37 & 48,5 & 26 & 28,1 & 31,2 & 31,2 & 21,7 & 21,4 \\
\hline $4-3$ & 41,1 & 50,2 & 51,4 & 28,3 & 25,4 & 27,4 & 28 & 23,4 & 21,5 \\
\hline $12-3$ & 49,4 & 45,5 & 42,9 & 31,2 & 20,6 & 21,6 & 15,2 & 21,4 & 26 \\
\hline $20-3$ & 50 & 50,4 & 49 & 20,8 & 22,2 & 21,2 & 15,8 & 17,1 & 17 \\
\hline $28-3$ & 43,4 & 20,7 & 23,5 & 14,4 & 15,2 & 21,1 & 16,7 & 17,1 & 17,2 \\
\hline $7-4$ & 35 & 34 & 36 & 26,1 & 17,2 & 18,9 & 15,3 & 7,9 & 10,1 \\
\hline $16-4$ & 34,5 & 31,7 & 27 & 26,1 & 22,6 & 17,2 & 17,8 & 12,5 & 13,1 \\
\hline $26-4$ & 31,3 & 26,4 & $2.0,5$ & 28,8 & 25,5 & 23,6 & 20,1 & 18,6 & 13,8 \\
\hline
\end{tabular}

d: densité (individus $\left./ \mathrm{m}^{2}\right) ; \mathrm{N}$ : dose d'azote $(\mathrm{Kg} / \mathrm{ha}) ; \mathrm{d}_{1}: 150$ individus $/ \mathrm{m}^{2} ; d_{2}: 300$ individus $/ \mathrm{m}^{2} ; d_{3}: 450$ individus $/ \mathrm{m}^{2}$.

Tableau IIl. Répartition de l'énergie interceptée par la vesce et par strates, en \% de l'énergie incidente. IFV: indice foliaire de la vesce; $\mathrm{R}_{\mathrm{iv}}$ : rayonnement intercepté par la vesce.

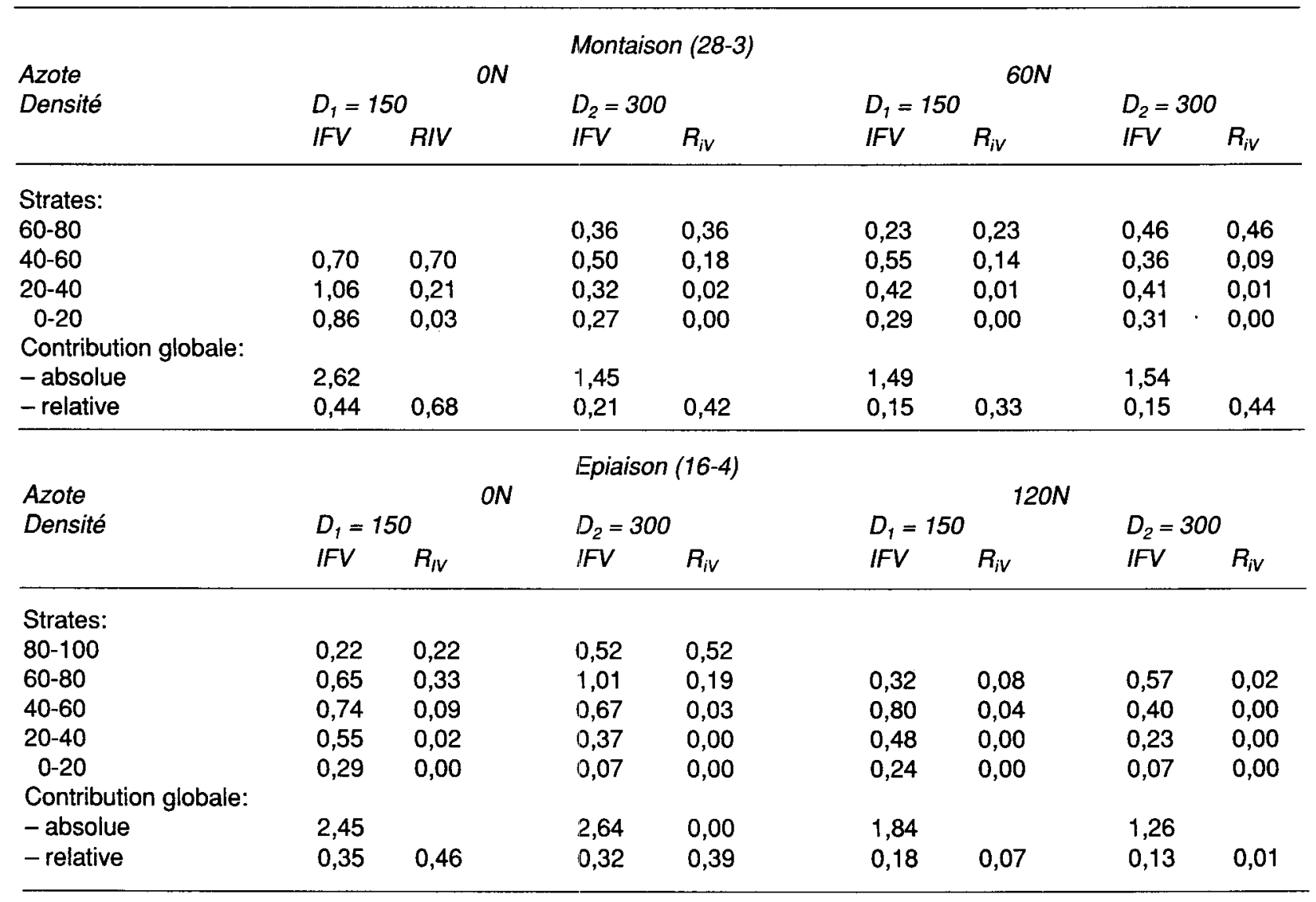

$-R_{i}=$ rayonnement intercepté,

- $\mathrm{IF}_{\mathrm{s}}=$ indice foliaire cumulé au-dessus de la strate $\mathrm{s}$,

$-K=$ coefficient d'extinction caractéristique du couvert.

En l'absence de données sur les caractéristiques du couvert (orientation des feuilles, réflec- tance) et sur la variation de $\mathrm{K}$ suivant la densité (Joggi, 1977), on a retenu pour $K$ une valeur constante de 0,70 (Brougham, 1960).

D'une manière générale, on peut constater qu'à la montaison la vesce contribue beaucoup plus, en valeur relative, à l'interception de l'énergie que pourrait le faire penser la seule considé- 
ration de l'indice de surface foliaire. Il en est de même, mais dans une moindre mesure, à l'épiaison, en l'absence d'apport d'azote. En revanche, l'apport de 120 unités d'azote inverse la situation: l'indice de surface foliaire de la vesce surestime alors sa contribution au couvert par rapport au rayonnement qu'elle intercepte, et cela d'autant plus que la densité est élevée.

Ces résultats expliquent en partie la variation dans le temps de la liaison entre biomasse accumulée et indice de surface foliaire.

Jusqu'au stade d'épiaison, la biomasse aérienne produite dans les peuplements associés est corrélée à l'indice foliaire global. A faible peuplement, les coefficients de corrélations obtenus sont de: $r=0,88 ; 0,87$ et 0,93 respectivement pour 0,60 et $120 \mathrm{~N}$, et les pentes des droites sont, dans le même ordre, de: $b=3,84 ; 2,87$ et 3,51 , pour $n=27$ couples.

En peuplement moyen $\left(d_{2}=300\right.$ ind. $\left./ \mathrm{m}^{2}\right)$ les coefficients de corrélation diminuent avec le niveau d'azote: $r=0,88 ; 0,61$ et 0,73 et les pentes de droites sont de: $b=5,52 ; 5,45$ et 4,54 respectivement pour $\mathrm{N} 1$, N2 et $\mathrm{N} 3$ pour le même nombre de couples que ci-dessus. Au-delà du stade d'épiaison, la liaison semble dépendre d'autres paramètres que l'indice foliaire. L'indice foliaire de l'association peut donc être utilisé pour l'étude de la biomasse aux stades antérieurs à l'épiaison.

\section{Conclusion}

L'analyse de la dynamique de croissance, chez les 2 espèces, montre évidemment que la biomasse est fortement corrélée à la surface foliaire développée par la plante. II en est de même pour le poids spécifique des feuilles qui est à son tour corrélé à la matière sèche produite.

Le paramètre surface foliaire a réagi différemment chez les 2 espèces aux facteurs: densités et fertilisation azotée. La fertilisation azotée, ayant augmenté la croissance de l'avoine, a excercé un effet dépressif sur la croissance de la vesce par l'ombrage qu'elle crée. Ces résultats sont à rapprocher de ceux de Cruz et Lemaire (1986), qui ont rapporté que tout facteur contribuant à ralentir préférentiellement la vitesse d'établissement de la surface foliaire de l'une des 2 espèces aura pour conséquence une augmentation de la réponse à l'azote de l'autre espèce dans l'association.

II ressort donc de cela que la vesce souffre d'un manque de disponibilités en lumière. Des indices foliaires, en association, supérieurs à ceux des cultures pures, d'une part, et la contribution de la vesce dans cet indice total, qui est d'autant plus faible que la fertilisation azotée est élevée pour une densité donnée à un stade donné, d'autre part, suggèrent une compétition pour la lumière. Cette compétition a été confirmée par l'analyse de la structure des profils de surface foliaire établis pour certains stades. En effet, dans toutes les densités testées, la vesce en association, pour échapper à la contrainte exercée par son partenaire, s'étiole davantage en occupant les strates supérieures, où l'avoine est absente ou peu présente, c'est-à-dire les mieux éclairées. Bien entendu, cet étiolement n'est pas sans conséquences sur les individus de vesce. II se fait au détriment de la richesse en feuilles dont dépend la teneur en azote total (Ouknider et Jacquard, 1986). Pour un peuplement donné $\left(d_{1}, d_{2}\right.$ ou $\left.d_{3}\right)$, la densité de la strate augmente avec la fertilisation azotée.

La structure des profils de surface foliaire se modifie avec l'âge des plantes. Au stade d'épiaison de la graminée, la densité foliaire dans les couches sub-inférieures $(20-40 \mathrm{~cm})$ et moyenne $(40-60 \mathrm{~cm})$ est très élevée, au point que l'on assiste à une situation de dépérissement total des feuilles de la couche $0-20 \mathrm{~cm}$ pour les peuplements $d_{2}$ et $d_{3}$ : pour les 2 espèces, la surface foliaire dans cette strate est nulle. II en résulte donc que la compétition pour la lumière est non seulement déterminée par la contribution de chaque espèce à l'indice foliaire global, mais aussi par la position des feuilles dans les différentes strates du couvert végétal. La combinaison de ces 2 critères permet d'estimer la participation des 2 espèces à l'interception de l'énergie, car c'est en définitive cette participation qui détermine la croissance relative des 2 espèces.

Cependant, jusqu'au stade d'épiaison de l'avoine, la biomasse produite par les peuplements associés est corrélée à l'indice foliaire global. Ce dernier semble être un outil intéressant pour déterminer l'intensité de la compétition pour la lumière. Comme ceci a été remarqué par certains auteurs, l'intensité de la compétition pour la lumière n'est pas liée directement à la croissance en matière sèche des 2 espèces, mais à leur capacité d'interception de la lumière, laquelle est déterminée à la fois par la dynamique d'expansion de l'indice foliaire et par l'architecture du couvert végétal.

\section{Références}

Arnaud R. \& Niqueux M. (1982) Possibilités des associations du trèfle violet avec différentes graminées en moyenne montagne. Fourrages 89, 3-28

Broadbent F.E., Nakashima T. \& Chang G.Y. (1982) Estimation of nitrogen fixation by isotope dilution in field and greenhouse experiments. Agron. J., 74, 4, 625-628 
Brougham R.W. (1960) The relationship between the initial leaf area, total chlorophyll content, and maximum growth-rate of some pasture and crop plants. Ann. Bot. 24, 463-474

Brown R.H. \& Blaser R.E. (1968) Leaf area index in pasture growth. Herb. Abstr. 38, 1, 1-9

Chalamet A., Audergon J.M. \& Maitre J.P. (1983) Concurrence entre une graminée (Lolium multiflorum Lamk.) et une légumineuse (Trifolium pratense L.): utilisation du $\mathrm{N}^{15}$ pour l'étude de la nutrition azotée. Acta Oecol. Oecol. Plant. 4, 18, 125-133

Charles J.P. (1982) Utilisation en Suisse du trèfle violet et de la luzerne avec des graminées. Fourrages 90 , 39-56

Cruz P. (1985) Relation de compétition dans une association luzerne dactyle: étude des dynamiques de la croissance et des prélèvements d'azote. Thèse Doc. Ing. Univ. Paris-Sud.

Cruz P. \& Lemaire G. (1986) Analyse des relations de compétition dans une association de luzerne (Medicago sativa L.) et de dactyle (Dactylis glomerata L.) I. Effets sur les dynamiques de croissance en matière sèche. Agronomie, 6(8), 727-734

Cruz P. et Lemaire G. (1986) Analyse des relations de compétition dans une association de luzerne (Medicago sativa L.) et de dactyle (Dactylis glomerata L.). II. Effets sur la nutrition azotée des deux espèces. Agronomie 6(8) 735-742

Doberti H., 1974. Association avena-vicia como forrage supplementario en Magallanes. Agric. Tec. 32., 1, 20-23

Donald C.M. (1963) Competition among crop and pasture plants. Adv. agron. 15,1-118

Faris M.A. \& Ta T.C. (1985) Study of nitrogen transfer from alfalfa to associated timothy under field conditions. In: Proceedings of the 15th International Grassland Congress, Kyoto, Japan. 24-31 August 1985, pp. 448-449

Gosse G., Chartier M. \& Lemaire G. (1984) Mise au point d'un modèle de prévision de production pour une culture de luzerne. C.R. Acad. Sci. Paris 298, Série III, $18,541-544$

Haynes J. (1980) Competitive aspects of the grasslegume association. Adv. Agron. 33, 227-261

Ismaili M. \& Weaver R.W. (1986) Competition between Siratro and Kleingrass for $15 \mathrm{~N}$ labelled minerallized nitrogen. Plant Soil 96, 327-335

Jacquard P. (1977) Relations entre espèces dans les associations graminées-légumineuses Sel. Fr. 24, 3-28

Joggi D. (1977) Photosynthèse et formation du rende- ment de cultures de Trifolium pratense $L$. en fonction de la structure du couvert. Thèse Doc. Sciences Techniques, Ecole polytechnique fédérale, Zürich

Laissus R. (1979) Associations graminées-trèfle blanc. Sel. Fr. 27, 45-48

Lemaire G. \& Salette J. (1984) Relations entre dynamique de prélèvement d'azote pour un peuplement de graminées fourragères. I. Etude de l'effet du milieu. Agronomie, 4 (5), 423-430

Lemaire G. \& Salette J. (1984) Relations entre dynamique de prélèvement d'azote pour un peuplement de graminées fourragères. II. Etude de la variabilité entre génotypes. Agronomie 4, 5, 431-436

Lescure C., Chalamet A. \& Maitre J.P. (1986) Transfert direct d'azote dans l'association légumineuse (Trifolium pratense L.) - graminée (Lolium italicum Lamk.) problèmes soulevés dans son estimation par ${ }^{15 \mathrm{~N}}$. Acta Oecol. Oecol. Plant. 7, 21, 2, 213-217

Martin MPLD \& Snaydon R.W. (1982) Root and shoot interaction between barley and field beans when intercropped. J. Appl. Ecol. 19, 263-272

Ouknider M. \& Jacquard P. (1986) Production de matière sèche et valeur nutritive de l'association vesce-avoine en zone méditerranéenne. Fourrages 105, 39-62

Ouknider M. \& Jacquard P. (1988) Un modèle d'association graminée-légumineuse: le mélange vesce (Vicia sativa L.) - avoine (Avena sativa L.). Agronomie 8(2) 97-106

Plancquaert P. (1976) Résultats expérimentaux de I'ITCF concernant les associations graminées légumineuses. Fourrages 66, 31-62

Salette J. \& Lemaire G. (1981) Sur la variation de la teneur en azote des graminées fourragères pendant leur croissance: formulation d'une loi de dilution. C. $R$. Acad. Sci. Paris 292, 875-878

Ta T.C. \& Faris M.A. (1987) Species variation in the fixation and transfer of nitrogen from legumes to associated grasses. Plant Soil 98, 265-274

Valis I. (1978) Nitrogen relationships in grass/legume mixture, 190-201. In: Plant Relations in Pastures. CSIRO, J.R. Wilson, Canberra

Wacquant J.P., El-Chahatha H. \& Jacquard P. (1981) Effect of mineral stress on competition and associated growth of a grass (Lolium italicum Lamk.) and a legume (Trifolium pratense L.). Proceedings of the symposium of Nottingham. (E.E. Wright, ed.) Brit. Grassland Soc., Maidenhead U.K., pp. 231-234

Wery J. (1983) Contribution à l'étude de la nutrition azotée d'une légumineuse fourragère (Medicago sativa L.) et de légumineuses à graines. Thèse Doc. Ing. USTL, Montpellier 